\title{
Clinical Manifestation and Incidence of Cardiopulmonary Complications in Early Systemic Sclerosis Patients with Different Antibody Profiles
}

\author{
Suparaporn Wangkaewa, d, Narawudt Prasertwittayakij ${ }^{b}$, Juntima Euathrongchit ${ }^{\mathrm{c}}$
}

\begin{abstract}
Background: There has been no prior inception cohort study comparing clinical manifestations and incidence rate (IR) of cardiopulmonary involvement among early systemic sclerosis (SSc) patients by difference in autoantibody profiles. We compared the differences in the clinical presentation at study entry and cumulative organ complications at last visit, as well as the IR of cardiopulmonary complications between anti-topoisomerase I antibody-positive SSc patients (pATA), ATA-negative (nATA), and the positive anti-centromere antibody patients (pACA).
\end{abstract}

Methods: An inception cohort of early diagnosis SSc patients (disease duration $\leq 3$ years) seen at the Rheumatology Clinic, Maharaj Nakorn Chiang Mai Hospital, between January 2010 and June 2016, was studied. SSc patients who had follow-up duration as less than 1 year and those diagnosed with an overlap syndrome were excluded. All participants underwent electrocardiography (ECG), echocardiography, and high-resolution computed tomography (HRCT) at the study entry and then annually.

Results: A total of 114 patients (90 diffuse cutaneous SSc (dcSSc), 69 women) with mean (standard deviation, SD) disease duration of 11.7 (8.8) months at cohort entry and an observational period of 3.8 (1.6) years, were recruited. There were 89 patients $(78.1 \%)$ with pATA, 18 (15.8\%) with nATA, and 7 (6.1\%) with pACA. At enrollment, both pATA and nATA groups had a higher prevalence of dcSSc subtype, and interstitial lung disease (ILD) when compared with the pACA group. At the last visit, the pATA group had a higher cumulative prevalence of digital ulcers, joint contracture and tendon friction rub than the other groups. Both the pATA and nATA groups had a significantly

Manuscript submitted April 20, 2019, accepted May 13, 2019

a Division of Rheumatology, Department of Internal Medicine, Faculty of Medicine, Chiang Mai University, Chiang Mai, Thailand

${ }^{b}$ Division of Cardiology, Department of Internal Medicine, Faculty of Medicine, Chiang Mai University, Chiang Mai, Thailand

'Division of Diagnostic Radiology, Department of Radiology, Faculty of Medicine, Chiang Mai University, Chiang Mai, Thailand

${ }^{\mathrm{d} C}$ Corresponding Author: Suparaporn Wangkaew, Division of Rheumatology, Department of Internal Medicine, Faculty of Medicine, Chiang Mai University, Chiang Mai 50200, Thailand. Email: suparaporn.w@cmu.ac.th

doi: https://doi.org/10.14740/jocmr3849 higher IR of ILD compared to the pACA group (54.9 and 57.8 vs. 6.3 per 100 person-years). During the study period, no suspected myositis, systolic pulmonary artery pressure (sPAP) $\geq 50 \mathrm{~mm} \mathrm{Hg}$ or cardiac complications was observed in the pACA group.

Conclusions: In our study cohort, the majority of which were dcSSc subtype with pATA, it was found that the presence of SSc-specific autoantibodies was associated with a distinctive clinical presentation and cumulative internal organ involvement, even in the early phase of the disease. Cardiopulmonary complications were rarely seen in the pACA group; whereas ILD complications were very common in both the pATA and nATA groups. A further study into the association of autoantibodies in nATA patients with ILD complications is needed.

Keywords: Anti-topoisomerase I antibody; Anti-centromere antibody; Clinical manifestation; Incidence; Systemic sclerosis

\section{Introduction}

Systemic sclerosis (SSc) is a complex autoimmune connective tissue disease of unknown etiology which is contributed by vascular injury, autoimmune alteration and fibrosis of the skin and visceral organs. SSc patients are mainly classified into diffuse cutaneous SSc (dcSSc) and limited cutaneous SSc (1cSSc) subsets according to the extent of skin involvement. Although dcSSc and lcSSc subsets are associated with organ manifestations, large cohort studies have shown that autoantibody status in SSc patients is also associated with clinical manifestations and organ system involvement as well as prognostic and survival features [1-4]. Information from both clinical subset and autoantibody status should be combined to evaluate the longterm prognosis of SSc patients [2].

Anti-topoisomerase I antibody (ATA) and anti-centromere antibody (ACA) are the autoantibodies routinely used in general clinical practice for early diagnosis of SSc according to the 2013 American College of Rheumatology (ACR)/the European League Against Rheumatism (EULAR) classification criteria for SSc [5]. Prior large prevalence studies have reported that there is a higher prevalence of the dcSSc subset $[2,3,6-8]$, joint involvement $[2,3]$, tendon friction rub [2, $3]$, digital ulcers [2], interstitial lung disease (ILD) [2, 3, 68], pulmonary hypertension associated with ILD [2] and heart 
involvement $[2,3,6,7]$ in ATA-positive SSc patients than in the ACA-positive subset. On the other hand, there is a higher prevalence of the lcSSc subset $[2,3,6]$ and isolated pulmonary hypertension $[2,3]$ in the ACA-positive patients. Therefore, the ATA-positive subset has been associated with more severe organ involvement $[2,3,6-8]$ and poorer clinical outcome than the ACA-positive subset $[1,3]$.

Most reports which have pointed out comparisons between different autoantibody profiles regarding the prevalence of clinical manifestations have included SSc patients with variation in the phase of the course of the disease. Moreover, it has been reported that Thai SSc patients have a higher prevalence of an ATA-positive test (80-85.7\%) [9, 10] than those from East Asia (Japan (23.5\%) [7] and China (59.9\%) [8]), and Western countries $(22.2-36.8 \%)$ [3, 11]. Therefore, the difference seen in Thailand as regards the characteristics of autoantibody profiles may affect the clinical presentation and incidence of cardiopulmonary complications when compared to the earlier studies $[3,7,8,11]$.

In addition, there is no prior inception cohort study comparing clinical manifestations and incidence rate (IR) of cardiopulmonary involvement among SSc patients by difference in autoantibody profiles. Therefore, this inception cohort study was conducted to compare the differences in the initial clinical presentation and cumulative organ involvement, as well as the IR of cardiopulmonary complications between ATA-positive SSc patients, ATA-negative and ACA-positive SSc patients.

\section{Materials and Methods}

\section{Study population}

This study cohort was recruited from the Rheumatology Clinic, Chiang Mai University, and included all consenting, consecutive adults ( $\geq 18$ years). The cohort consisted of early diagnosed SSc patients (disease duration $\leq 3$ years from first non-Raynaud's phenomenon (NRP) attributable to SSc), from January 2010 to June 2016. All patients fulfilled the 1980 classification criteria of SSc [12] and/or the ACR/EULAR criteria 2013 for the classification of SSc [5]. The patients were classified as dcSSc or lcSSc in accordance with LeRoy and Medsger's classification criteria [13]. Exclusion criteria were SSc patients who had follow-up duration from the enrolment on the study to their last visit as less than 1 year and those diagnosed with an overlap syndrome (SSc with systemic lupus erythematosus or SSc with rheumatoid arthritis).

\section{Clinical evaluation}

At cohort entry the following were recorded: demographic data, clinical presentation, and current medication. In addition laboratory tests were carried out including complete blood count, creatinine, creatine kinase (CK) levels, erythrocyte sedimentation rate (ESR), and prohormone of brain natriuretic peptide (proBNP) level. Tests for antinuclear antibodies and ACAs (by immunofluorescence on Hep2 cells), and ATA (by enzyme-linked immunosorbent assay (ELISA)) were also carried out. The severity of the skin involvement was assessed using a modified Rodnan skin score (mRSS) [14]. All participants underwent electrocardiography (ECG), echocardiography, and high-resolution computed tomography (HRCT) at the study entry and then annually. The ECG and echocardiography results were assessed by an experienced cardiologist (N. P.) blinded to the clinical data. The HRCT results were interpreted by an experienced thoracic radiologist (J. E.) blinded to the clinical data. The patients were seen at regular intervals between 1 and 3 months based on the severity of their disease and they received medical treatment as recommended by their attending rheumatologists.

Complete data, including clinical manifestations and blood tests, were collected every 6 months. At the last visit, the cumulative clinical manifestations were recorded. The survival status of the participants was recorded for all patients in June 2016.

\section{Definitions}

The onset of SSc was defined as the time of the first NRP attributable to SSc as reported by the patient. Duration of disease was calculated as the interval between the disease onset and the time at the study entry. Duration of follow-up was calculated as the interval between the cohort entry and the time of the last follow-up or death. Initial clinical characteristics were defined as the organ involvement detected at cohort entry. $\mathrm{Cu}-$ mulative clinical characteristic was defined as the presence of organ involvement being more than one in the recorded data during the observational period.

The presence of organ involvement was assessed as "yes" or "no" according to the following definitions: peripheral vascular manifestations were defined by the presence of Raynaud's phenomenon (RP), digital pitting scar, digital ulcer or telangiectasia; a digital ulcer was defined as active or healed ulceration which was present at the volar aspect of the digital pulp.

Skin manifestation was defined by the presence of hypohyperpigmentation. Musculoskeletal manifestations were defined by the presence of arthritis, joint contracture, tendon friction rub, or suspected myositis. Joint contracture was defined as presence of stiffness in any joints, which decreased the range of motion and prevented full extension. Suspected myositis was defined as CK levels elevated to be $\geq 500 \mathrm{IU} / \mathrm{L}$ in the absence of other explainable causes such as statin use or hypothyroidism.

Gastrointestinal involvements were defined as the presence of gastroesophageal reflux disease (GERD) or dysphagia symptoms reported by the patient.

Cardiac involvements were defined by the presence of any of the following which were detected by echocardiography, in the absence of other explainable causes: 1) pericardial effusion; 2) left ventricular ejection fraction lower than $50 \%$ (LVEF < 50\%); or 3) right ventricular dysfunction (RVD). RVD was defined as being present when the tricuspid annular plane systolic excursion (TAPSE) was less than $1.6 \mathrm{~cm}$, the right ventricular annular velocity was less than $10 \mathrm{~cm} / \mathrm{s}$, the 
right ventricular fraction area change was less than $35 \%$, or a localized right ventricular aneurysmal change was detected [15]. In addition, cardiac involvement was also defined as being existed if presence of conduction alteration was detected by ECG, which includes any bundle branch block, second or third degree atrioventricular block, QRS duration (QRSd) > $120 \mathrm{~ms}$, or prolonged corrected QT (QTc) interval [16]. The prevalence of left ventricular diastolic dysfunction prior to 2016 was based on the 2009 recommendations for evaluation of left ventricular diastolic dysfunction by echocardiography [17]. Since 2016, the diagnosis is based on the 2016 recommendations for the evaluation of left ventricular diastolic function by echocardiography [18].

Pulmonary involvement was defined as the presence of: 1) ILD determined by HRCT, or 2) suspected pulmonary arterial hypertension defined as estimated systolic pulmonary arterial pressure (sPAP) being $\geq 50 \mathrm{~mm} \mathrm{Hg}$ at rest, with normal LVEF determined by echocardiography [19].

Scleroderma renal crisis was defined as present if fulfilling the criteria described by the international scleroderma crisis study group [20].

Sicca symptoms were defined as present when ocular or oral dryness was reported by the patients.

\section{Statistical analysis}

The descriptive data are presented as percentages or mean \pm $\mathrm{SD}$, where appropriate. Clinical manifestations and laboratory investigations were compared between groups based on the three serological profiles (single ATA-positive (ATA-positive), both ATA and ACA-negative (ATA-negative) and single ACApositive (ACA-positive)), using Chi-square test, Fisher's exact test, one-way analysis of variance (ANOVA), and KruskalWallis test as appropriate. The Mann-Whitney U test was used to compare nonparametric continuous variables between two subgroups. The time from first NRP to the first diagnosed LVEF $<50 \%$, RVD, ILD and sPAP $\geq 50 \mathrm{~mm} \mathrm{Hg}$ was analyzed using the Kaplan-Meier plot. The patient data were censored when: 1) any of the following cardiopulmonary involvements were detected: $\mathrm{LVEF}<50 \%$, RVD, ILD, or sPAP $\geq 50 \mathrm{~mm} \mathrm{Hg}$; and 2) any event occurred which included death or the end of the study was reached.

The comparison of the differences in cumulative incidence consisted of analysis of LVEF $<50 \%$, RVD, ILD, and sPAP $\geq 50 \mathrm{~mm} \mathrm{Hg}$ between the three serology subgroups, using the log-rank test. The IR of any cardiopulmonary involvement between the two groups was compared using the Mantel Haenszel method. $\mathrm{P}$ values $<0.05$ were considered statistically significant. Statistical analyses were performed using Stata for Windows version 13.0 (StataCorp, College Station, TX, USA).

\section{Ethics statement}

This study was approved by the Chiang Mai University Research Ethics Committee in compliance with the 1964 Declaration of Helsinki and its later amendment. All patients gave written consent at study entry.

\section{Results}

\section{Demographic and clinical characteristics}

Out of the 115 early-SSc patients which fulfilled the inclusion criteria and were initially enrolled into the study, one patient with dcSSc was excluded due to having a combined ATA-positive and ACA-positive test, leaving a cohort of 114 patients (69 women, $90 \mathrm{dcSSc}$ ) in the final analysis. Of the 114, there were 89 patients $(78.1 \%)$ who were ATA-positive, 18 (15.8\%) ATAnegative and seven (6.1\%) ACA-positive. One hundred twelve patients $(98.2 \%)$ had an ANA-positive result (titer $\geq 1: 160)$, of which $71(63.4 \%)$ had positive patterns as fine speckle and nucleolar, $13(11.6 \%)$ coarse speckle, eight (7.1\%) fine speckle, eight $(7.1 \%)$ homogeneous, seven $(6.3 \%)$ centromere, three $(2.7 \%)$ homogeneous and nucleolar and two (1.7\%) nucleolar. The mean \pm SD age at disease onset was $51.4 \pm 8.5$ years and the duration of the disease from the first NRP to the study entry was $11.7 \pm 8.8$ months. The mean \pm SD duration of follow-up from the study entry was $3.8 \pm 1.6$ years.

Demographic data between the three serology subgroupings at cohort entry were compared. The results are shown in Table 1. The ATA-positive group had a significantly higher prevalence dcSSc subtype compared to patients in the ATAnegative and ACA-positive groups. The ACA-positive subgroup had significantly longer mean disease duration from NRP to study entry compared to the ATA-positive and ATAnegative groups.

There were no significant differences between the three serology subgroups with regard to age at disease onset, and duration of follow-up, as well as the proportion of presenting manifestation, and immunosuppressant or corticosteroid use. The mean $\pm \mathrm{SD}$ dose of prednisolone was $3.0 \pm 4.1 \mathrm{mg} /$ day. At the end of the study, 87 patients $(76.3 \%)$ continued followup, $13(11.4 \%)$ referred themselves to other hospitals due to clinical improvement, eight (7.0\%) failed to follow-up, and six $(5.3 \%)$ patients died.

\section{Comparison of clinical manifestations between serology subgroupings at cohort entry}

At cohort entry, the ACA-positive group had a significantly higher prevalence of telangiectasia than the ATA-positive and ATA-negative groups. The ATA-positive group had a significantly higher prevalence of skin hypo-hyperpigmentation than the ATA-negative and ACA-positive groups. Both ATA-positive and ATA-negative groups showed a significantly higher prevalence of ILD compared to the ACA-positive group ( $80.9 \%$ and $77.8 \%$ vs. 0 ) (Table 2$)$. Analysis of the initial laboratory tests showed that there were no significant differences between the three subgroupings (ATA-positive, ATA-negative and ACA-positive) with regard to values of creatinine, $\mathrm{CK}$ and proBNP.

According to the initial echocardiography, there were no 
Table 1. Demographics

\begin{tabular}{|c|c|c|c|c|}
\hline & ATA positive $(n=89)$ & ATA negative $(\mathrm{n}=18)$ & ACA positive $(n=7)$ & P value \\
\hline DcSSc subtype & $79(88.8)^{\mathrm{a}, \mathrm{c}}$ & $9(50.0)$ & $2(28.6)$ & $<0.001$ \\
\hline Female & $50(56.2)$ & $14(77.8)$ & $5(71.4)$ & 0.206 \\
\hline Disease duration (months) & $11.7 \pm 8.9^{\mathrm{c}}$ & $8.1 \pm 4.6^{b}$ & $21.4 \pm 8.9$ & 0.003 \\
\hline Follow-up duration (years) & $3.9 \pm 1.6$ & $3.5 \pm 1.4$ & $3.4 \pm 1.5$ & 0.466 \\
\hline Raynaud's phenomenon & $12(13.5)$ & $6(33.3)$ & $1(14.3)$ & 0.120 \\
\hline Swollen or indurated skin & $18(20.2)$ & $3(16.7)$ & 0 & 0.666 \\
\hline Arthritis & $6(6.7)$ & $1(5.6)$ & 0 & 1.000 \\
\hline Combined manifestation & $49(55.1)$ & $7(38.9)$ & $6(85.7)$ & 0.120 \\
\hline \multicolumn{5}{|l|}{ Current medication } \\
\hline
\end{tabular}

Data are expressed as mean \pm SD or number (\%); dcSSc: diffuse cutaneous systemic sclerosis; in total group, immunosuppressive medication included: 30 cyclophosphamide (26.3\%), 14 methotrexate (12.3\%), five azathioprine (4.4\%), and three mycophenolate mofetil (2.6\%). aP < 0.05 , ATA positive versus ATA negative; ${ }^{b} \mathrm{P}<0.05$, ATA negative versus ACA positive; ${ }^{\mathrm{c}} \mathrm{P}<0.05$, ATA positive versus ACA positive.

significant differences between the three groups regarding the mean \pm SD values of $\%$ LVEF $(68.4 \pm 7.9$ vs. $66.7 \pm 7.2$ vs. $70.2 \pm 4.5, \mathrm{P}=0.562)$, tricuspid regurgitation velocity $(\mathrm{m} / \mathrm{s})$ $(2.4 \pm 0.3$ vs. $2.6 \pm 0.5$ vs. $2.5 \pm 0.3, \mathrm{P}=0.143)$, deceleration time (s) (0.22 \pm 0.05 vs. $0.21 \pm 0.05$ vs. $0.19 \pm 0.03, \mathrm{P}=0.184)$, and SPAP $(31.6 \pm 8.8$ vs. $36.5 \pm 15.5$ vs. $33.1 \pm 6.1, \mathrm{P}=0.230)$. In addition, the prevalence of left ventricular diastolic dysfunction was comparable between the three groups $(50.6 \% \mathrm{vs}$. $38.9 \%$ vs. $42.9 \%, \mathrm{P}=0.690)$.

\section{Comparison of cumulative clinical manifestations between serology subgroupings at the last visit}

At the last visit, the ATA-positive group had a higher cumulative clinical prevalence consisting of digital pitting scars, digital ulcers, telangiectasia, skin hypo-hyperpigmentation, joint contracture, and tendon friction rub than either the ATAnegative or ACA-positive group. In addition, both ATA-positive and ATA-negative groups had a higher cumulative proportion of ILD complications than the ACA-positive group (Table 3).

\section{Incidence of cardiopulmonary involvements}

After the cohort entry, with a mean observational period of 4 years, 14 patients $(12.3 \%)$ developed LVEF $<50 \%, 16(14.0 \%)$ developed RVD, 10 (8.8\%) developed ILD, five (4.4\%) developed sPAP $\geq 50 \mathrm{~mm} \mathrm{Hg}$, and six died (5.3\%).

Over the observational time from NRP, there were no significant differences between the three groups with regard to the cumulative incidence of LVEF $<50 \%(\mathrm{P}=0.271)$ (Fig. 1a), $\operatorname{RVD}(\mathrm{P}=0.200)$ (Fig. $1 \mathrm{~b})$ and $\mathrm{sPAP} \geq 50 \mathrm{~mm} \mathrm{Hg}(\mathrm{P}=0.223)$
(Fig. 1c). Interestingly, both the ATA-positive and ATA-negative groups showed significantly higher cumulative incidence of ILD compared to the ACA-positive group $(\mathrm{P}=0.006)$ (Fig. 1d). There were no cardiopulmonary complications including LVEF $<50 \%$, RVD and sPAP $\geq 50 \mathrm{~mm} \mathrm{Hg}$ observed in the ACA-positive group.

The IR of ILD complications per 100 person-years belonging to the ATA-positive, ATA-negative, and ACA-positive groups were $54.99,57.83$ and 6.33 , respectively. The IR ratio of ILD complications between ATA-positive and ACApositive groups was 8.68 (95\% confidence interval (CI) 2.32 72.98). In addition, the IR ratio of ILD complications between the ATA-negative and ACA-positive groups was 9.13 (95\% CI 2.15 - 81.87).

There was comparable modest IR between the ATA-positive and ATA-negative groups according to LVEF $<50 \%(3.83$ per 100 person-years vs. 1.36 per 100 person-years, $\mathrm{P}=0.319$ ), RVD (4.80 per 100 person-years vs. 1.40 per 100 person-years, $\mathrm{P}=0.202)$, and sPAP $\geq 50 \mathrm{~mm} \mathrm{Hg}$ (1.86 per 100 person-years vs. 4.60 per 100 person-years, $\mathrm{P}=0.223)$.

\section{Discussion}

To our knowledge, this is an inception cohort study in SSc patients in early stages of the disease (disease duration $\leq 3$ years) describing the association of clinical manifestations and IR of cardiopulmonary based on different autoantibody profiles routinely used in general practice. Our cohort included a homogeneous study population of SSc patients in early phases of the disease with a mean disease duration of 1 year from the first NRP to study entry.

This study population had a high prevalence in the dcSSc subset (78.9\%) and ATA-positive patients (78.1\%) which were 
Table 2. Comparison of Initial Clinical Manifestations by Immunological Profile at Cohort Entry

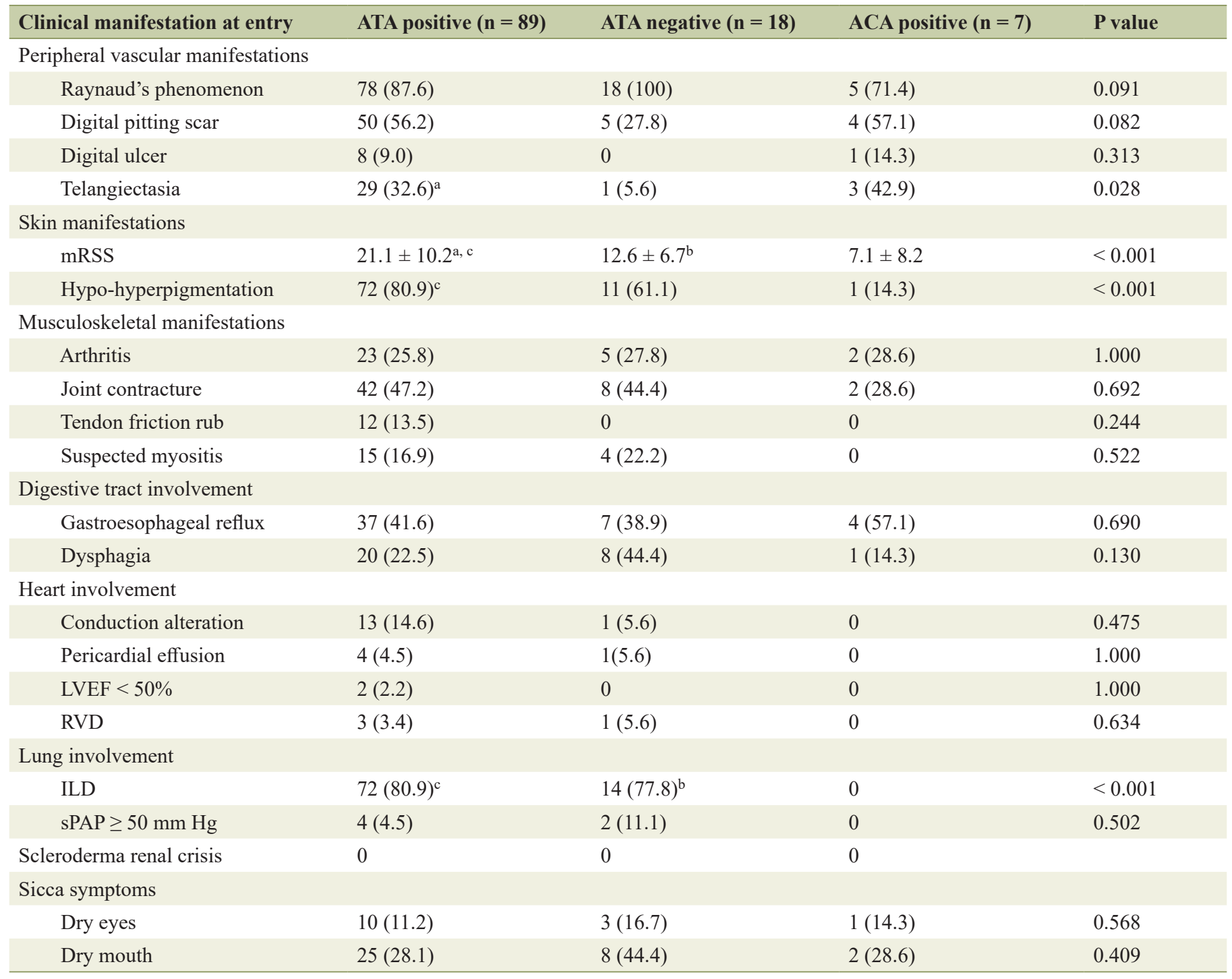

The data are presented as n (\%); LVEF: left ventricular ejection fraction; RVD: right ventricular dysfunction; ILD: interstitial lung disease; sPAP: estimated systolic pulmonary arterial pressure. ${ }^{a} \mathrm{P}<0.05$, ATA positive versus ATA negative; ${ }^{b} \mathrm{P}<0.05$, ATA negative versus ACA positive; ${ }^{\mathrm{c} P}<0.05$, ATA positive versus ACA positive.

higher than the 22.2-36.8\% range of ATA-positive patients reported by Western countries $[3,11]$, which should be highlighted. In addition in our study population, there was a low prevalence of ACA-positive patients (6.1\%) which is lower than the range of $20.3-32.3 \%$ reported by Western studies [3, 11]. Our study found that ATA-positive patients had a higher prevalence of the dcSSc subset $(88.8 \%)$ and had shorter disease duration from the first NRP to study entry, suggesting a rapidly progressive disease course compared to ACA-positive patients concordant with other studies [2-4].

At study entry, we found that ATA-positive patients had more severe mRSS and a higher proportion of hypo-hyperpigmentation, but had a lower proportion of telangiectasia than ACA-positive patients. In addition, ATA-negative SSc patients, of whom half were in the dcSSc subset, tended to have less severe clinical manifestations with respect to mRSS, telangiectasia and hypo-hyperpigmentation compared to ATApositive patients.

Interestingly, both ATA-positive and ATA-negative patients had a high comparable prevalence of ILD, whereas no ILD was observed in ACA-positive patients, suggesting both ATA-positive and ATA-negative SSc patients had more severe ILD complications than the ACA-positive patients. The nonspecific interstitial pneumonitis (NSIP) pattern was the most common HRCT finding in both ATA-positive (69.7\%) and ATA-negative subsets (72.7\%).

At the last visit, at the end of the mean observational period of 3.8 years, there were some differences in organ complications in the ATA-positive group. These consisted of a significantly higher cumulative prevalence of digital pitting 
Table 3. Comparison of Cumulative Clinical Manifestations by Immunological Profile at the Last Visit

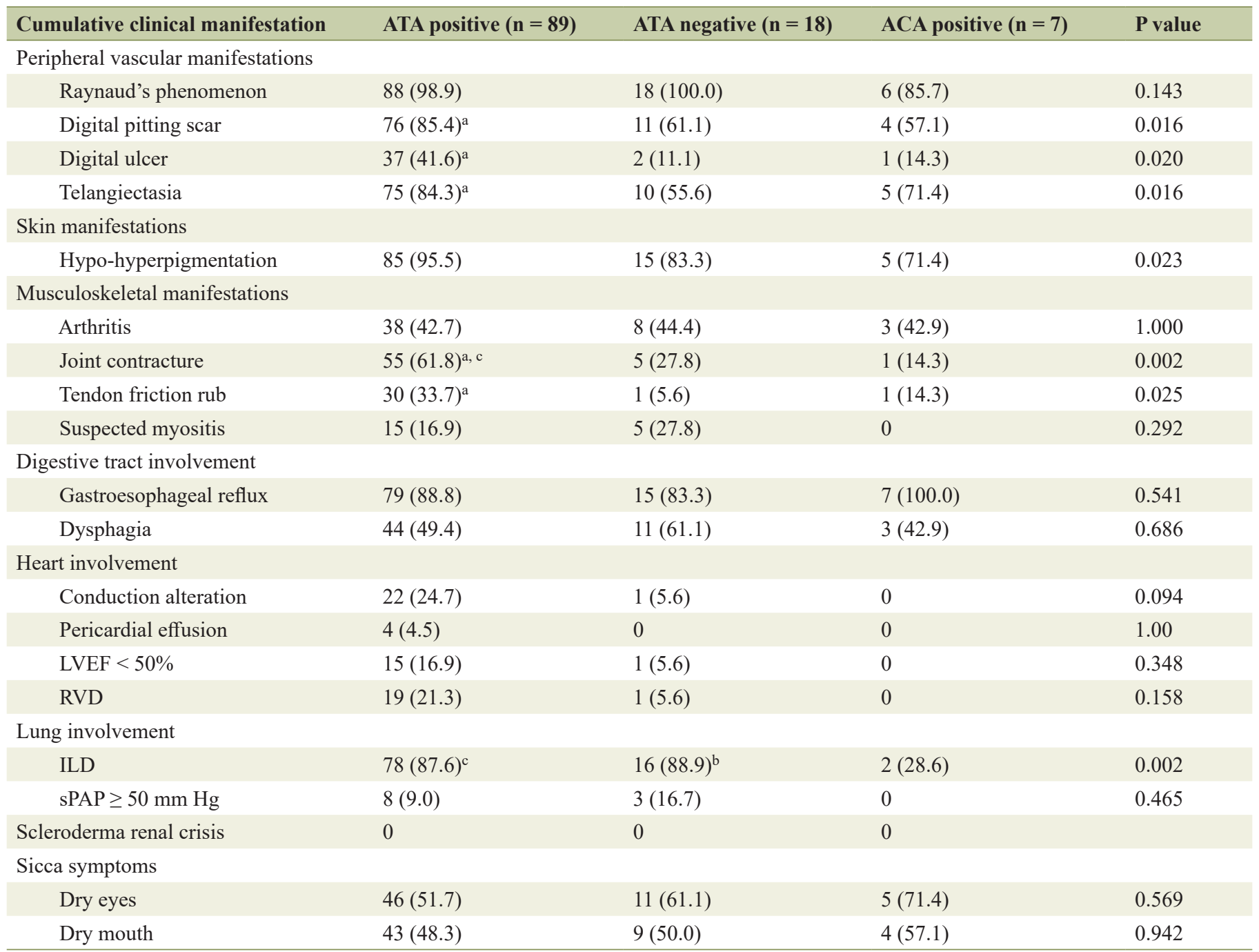

The data are presented as n (\%); LVEF: left ventricular ejection fraction; RVD: right ventricular dysfunction; ILD: interstitial lung disease; sPAP: estimated systolic pulmonary arterial pressure. ${ }^{a} \mathrm{P}<0.05$, ATA positive versus ATA negative; ${ }^{b} \mathrm{P}<0.05$, ATA negative versus ACA positive; ${ }^{\mathrm{c} P}<0.05$, ATA positive versus ACA positive.

scars, digital ulcers, telangiectasia, skin hypo-hyperpigmentation, joint contracture, tendon friction rub and ILD, suggesting more severe organ involvement and a more rapid progress of the disease even in the early phases, compared to the ATAnegative and ACA-positive groups. Similarly, data from other large studies supports the findings that ATA-positive patients had more severe cumulative organ complications than ACApositive patients $[2-4,6]$.

As regards cumulative prevalence of internal organ involvement, no significant differences were observed in the three serologic profile-related gastrointestinal, cardiac, and sicca symptoms, with findings concordant with Iniesta Arandia et al's report [4]. Contrary to this, Walker et al reported that cardiac involvement consisting of conduction block and diastolic dysfunction was more prevalent in an ATA-positive group than in an ACA-positive group [2]. The discrepancies regarding the prevalence of organ involvement based on the different serologic profiles might be attributable to differences in study population, definition of organ involvement, and follow-up duration. Our study included patients in the early phase of SSc with short duration of follow-up which differed from a prior study [2].

There was no suspected myositis or cardiac complications observed in ACA-positive SSc patients during the observational period. However, there was a significantly higher IR of ILD complications in both the ATA-positive and ATA-negative groups when compared to the ACA-positive group. Our study found modest IRs of $\% \mathrm{LVEF}<50 \%$, RVD, and $\mathrm{sPAP} \geq 50 \mathrm{~mm}$ $\mathrm{Hg}$ which were similar among the three autoantibody profiles. To our knowledge, data regarding the IR of these cardiopulmonary complications in early phase of SSc comparing between different antibody profiles is limited.

The major limitations of this study include the small sample size of early-SSc patients with a positive test for ACA. 

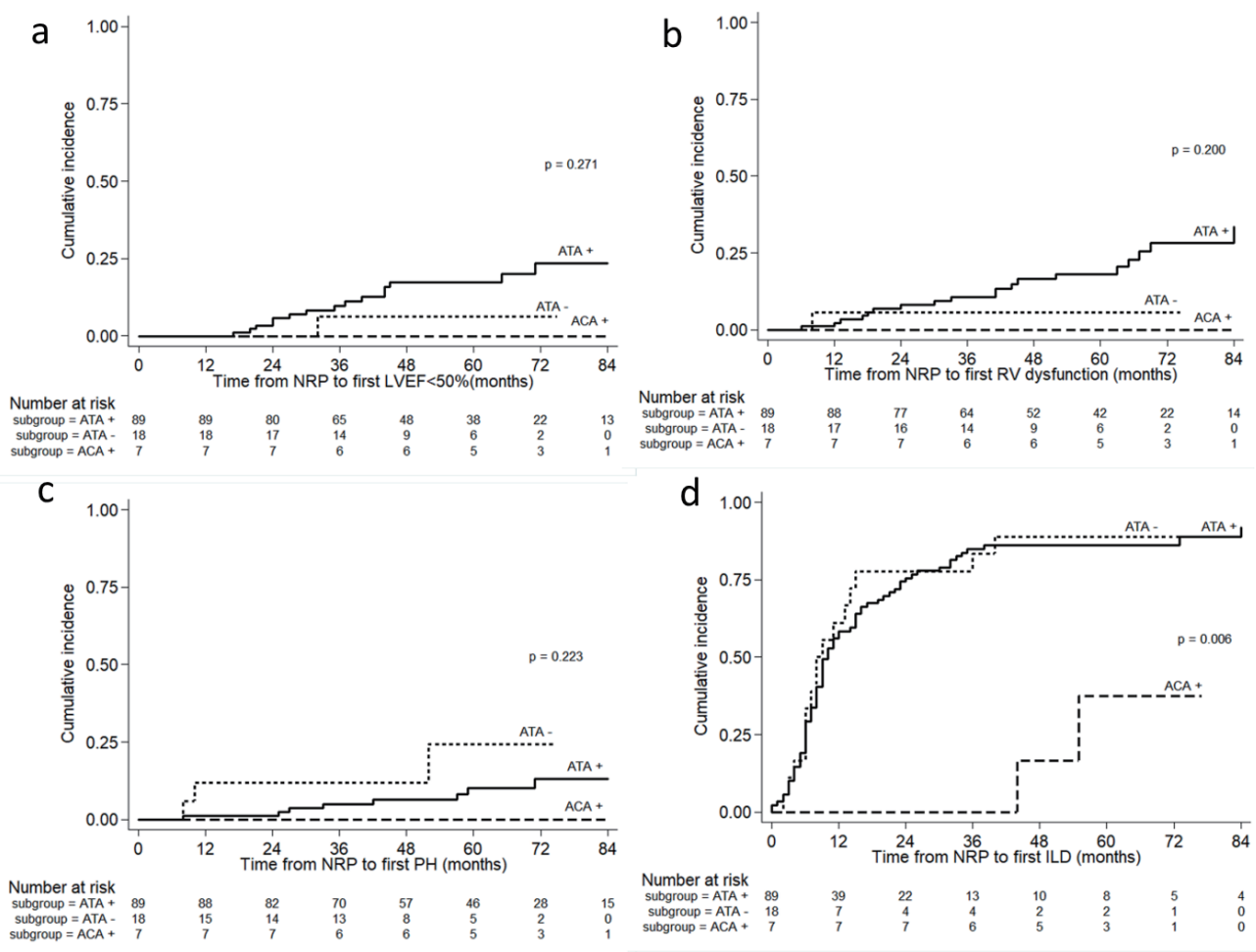

Figure 1. The cumulative incidence of (a) LVEF < 50\%, (b) RV dysfunction, (c) suspected PH, and (d) ILD in early SSc patients by different antibody profiles. The P value was from the log-rank test. LVEF: left ventricular ejection fraction; RV: right ventricular; $\mathrm{PH}$ : pulmonary arterial hypertension; ILD: interstitial lung disease.

In addition, further SSc-specific autoantibody testing for 18 ATA-negative SSc patients was not available during the study period. As the observational period was of short duration, with a mean observational period of 4 years from the study entry, our findings should be interpreted with caution. In addition we did not include early immunosuppressive treatment in our analysis due to the potential bias in the observational study. Another major limitation is that patients with suspected pulmonary arterial hypertension were not confirmed by right heart catheterization.

The strength of this study is that it is the first inception cohort study in early-SSc patients (disease duration $\leq 3$ years) to determine the difference in the clinical manifestations and IR of cardiopulmonary complications in a comparison based on routinely used autoantibody profiles in general clinical practice. An additional strength is that it is a single-center assessment with a limited number of investigators and uniform clinical, echocardiography, and HRCT assessments giving a consistency to the data collection and management.

\section{Conclusions}

In our study cohort, with majority of the patients being early dcSSc subtype who were ATA-positive, it was found that the presence of ATA or ACA is associated with distinctive presenting and cumulative organ involvement even in the early phase of disease in SSc patients. Cardiopulmonary complications were rarely seen in the ACA-positive group, whereas ILD complications were very common in both the ATA-positive and ATA-negative groups. A further study into the association of specific autoantibodies in ATA-negative patients with ILD complications is needed.

\section{Acknowledgments}

We would like to thank Mrs. Antika Wongthanee, medical statistician, for help with the statistical analysis.

\section{Financial Disclosure}

Authors have no financial disclosure to report.

\section{Conflict of Interest}

The authors declare that they have no conflict of interest concerning this article.

\section{Informed Consent}

All patients gave written consent at study entry. 


\section{Author Contributions}

SW designed the research; SW, NP and JE collected data; SW and AW analyzed data; SW wrote the paper. All authors read and approved the final paper.

\section{References}

1. Hesselstrand R, Scheja A, Shen GQ, Wiik A, Akesson A. The association of antinuclear antibodies with organ involvement and survival in systemic sclerosis. Rheumatology (Oxford). 2003;42(4):534-540.

2. Walker UA, Tyndall A, Czirjak L, Denton C, FargeBancel D, Kowal-Bielecka O, Muller-Ladner U, et al. Clinical risk assessment of organ manifestations in systemic sclerosis: a report from the EULAR Scleroderma Trials And Research group database. Ann Rheum Dis. 2007;66(6):754-763.

3. Steen VD. Autoantibodies in systemic sclerosis. Semin Arthritis Rheum. 2005;35(1):35-42.

4. Arandia NI, Simeon-Aznar CP, del Castillo AG, Arguelles DC, Rubio-Rivas M, Martinez LT, Hernandez FJG, et al. Influence of antibody profile in clinical features and prognosis in a cohort of Spanish patients with systemic sclerosis. Clin Exp Rheumatol. 2017;35 Suppl 106(4):98-105.

5. van den Hoogen F, Khanna D, Fransen J, Johnson SR, Baron M, Tyndall A, Matucci-Cerinic M, et al. 2013 classification criteria for systemic sclerosis: an American college of rheumatology/European league against rheumatism collaborative initiative. Ann Rheum Dis. 2013;72(11):1747-1755.

6. Denton CP, Krieg T, Guillevin L, Schwierin B, Rosenberg D, Silkey M, Zultak M, et al. Demographic, clinical and antibody characteristics of patients with digital ulcers in systemic sclerosis: data from the DUO Registry. Ann Rheum Dis. 2012;71(5):718-721.

7. Hashimoto A, Endo H, Kondo H, Hirohata S. Clinical features of 405 Japanese patients with systemic sclerosis. Mod Rheumatol. 2012;22(2):272-279.

8. Wang J, Assassi S, Guo G, Tu W, Wu W, Yang L, Xiao R, et al. Clinical and serological features of systemic sclerosis in a Chinese cohort. Clin Rheumatol. 2013;32(5):617621.

9. Foocharoen C, Suwannachat P, Netwijitpan S, Mahakkanukrauh A, Suwannaroj S, Nanagara R, Scleroderma Research G. Clinical differences between Thai systemic sclerosis patients with positive versus negative anti-topoisomerase I. Int J Rheum Dis. 2016;19(3):312-320.

10. Wangkaew S, Prasertwitayakij N, Phrommintikul A, Puntana S, Euathrongchit J. Causes of death, survival and risk factors of mortality in Thai patients with early systemic sclerosis: inception cohort study. Rheumatol Int. 2017;37(12):2087-2094.
11. Meier FM, Frommer KW, Dinser R, Walker UA, Czirjak L, Denton CP, Allanore Y, et al. Update on the profile of the EUSTAR cohort: an analysis of the EULAR Scleroderma Trials and Research group database. Ann Rheum Dis. 2012;71(8):1355-1360.

12. Preliminary criteria for the classification of systemic sclerosis (scleroderma). Subcommittee for scleroderma criteria of the American Rheumatism Association Diagnostic and Therapeutic Criteria Committee. Arthritis Rheum. 1980;23(5):581-590.

13. LeRoy EC, Black C, Fleischmajer R, Jablonska S, Krieg T, Medsger TA, Jr., Rowell N, et al. Scleroderma (systemic sclerosis): classification, subsets and pathogenesis. J Rheumatol. 1988;15(2):202-205.

14. Clements P, Lachenbruch P, Siebold J, White B, Weiner S, Martin R, Weinstein A, et al. Inter and intraobserver variability of total skin thickness score (modified Rodnan TSS) in systemic sclerosis. J Rheumatol. 1995;22(7):12811285.

15. Rudski LG, Lai WW, Afilalo J, Hua L, Handschumacher MD, Chandrasekaran K, Solomon SD, et al. Guidelines for the echocardiographic assessment of the right heart in adults: a report from the American Society of Echocardiography endorsed by the European Association of Echocardiography, a registered branch of the European Society of Cardiology, and the Canadian Society of Echocardiography. J Am Soc Echocardiogr. 2010;23(7):685-713; quiz 786-688.

16. Drew BJ, Ackerman MJ, Funk M, Gibler WB, Kligfield P, Menon V, Philippides GJ, et al. Prevention of torsade de pointes in hospital settings: a scientific statement from the American Heart Association and the American College of Cardiology Foundation. Circulation. 2010;121(8):10471060.

17. Nagueh SF, Appleton CP, Gillebert TC, Marino PN, Oh JK, Smiseth OA, Waggoner AD, et al. Recommendations for the evaluation of left ventricular diastolic function by echocardiography. J Am Soc Echocardiogr. 2009;22(2):107-133.

18. Nagueh SF, Smiseth OA, Appleton CP, Byrd BF, 3rd, Dokainish H, Edvardsen T, Flachskampf FA, et al. Recommendations for the evaluation of left ventricular diastolic function by echocardiography: an update from the American Society of Echocardiography and the European Association of Cardiovascular Imaging. J Am Soc Echocardiogr. 2016;29(4):277-314.

19. Galie N, Hoeper MM, Humbert M, Torbicki A, Vachiery JL, Barbera JA, Beghetti M et al. Guidelines for the diagnosis and treatment of pulmonary hypertension. Eur Respir J 2009;34(6):1219-1263.

20. Hudson M, Baron M, Tatibouet S, Furst DE, Khanna D, International Scleroderma Renal Crisis Study I. Exposure to ACE inhibitors prior to the onset of scleroderma renal crisis-results from the International Scleroderma Renal Crisis Survey. Semin Arthritis Rheum. 2014;43(5):666-672. 